\title{
Animés par la fièvre de la danse
}

par Myrella Roy

$\mathrm{L}$ a Société canadienne des pharmaciens d'hôpitaux (SCPH) a senfilé ses chaussures de danse pour vibrer aux rythmes de l'année 2012. Nous, les dirigeants, les bénévoles et les employées de la Société, avons fait de notre mieux pour épater les juges avec nos savantes pirouettes, de sorte que la SCPH soit proclamée le principal agent d'influence des progrès de la pratique en pharmacie hospitalière au Canada. Revivez les temps forts de l'interprétation de la SCPH et réfléchissez aux notes que vous nous accorderiez pour nos pas visionnaires, à savoir outiller les pharmaciens pour le plein exercice de leurs compétences; valoriser la profession; collaborer avec les parties prenantes essentielles; et favoriser la mobilisation des membres et leur réseautage.

Au cours de la dernière année, la SCPH s'est vue consacrée actrice incontournable sur la scène hip-hop des pénuries de médicaments. Nous avons poursuivi nos efforts pour créer un système national unique robuste pour la surveillance des pénuries de médicaments; nous avons accordé des entrevues à de nombreux médias; nous avons lancé un forum en ligne destiné aux discussions afférentes entre les membres de la SCPH; et nous avons comparu devant le Comité permanent de la santé de la Chambre des communes du Canada pour exprimer nos vues sur le rôle du gouvernement et de l'industrie dans la détermination de l'approvisionnement en médicaments au Canada (www.cshp.ca/advocacy/CSHPspeaks/drugShortages_e.asp). Nous nous sommes produits avec nos partenaires habituelsl'Association canadienne de la gestion de l'approvisionnement pharmaceutique (ACGAP), l'Association canadienne des chaînes de pharmacies (ACCP), l'Association canadienne du médicament générique (ACMG), l'Association des pharmaciens du Canada (APhC), l'Association médicale canadienne (AMC), BIOTECanada et les Compagnies de recherche pharmaceutique du Canada (Rx\&D)_pour tenir un atelier le 28 mai. Cet atelier, animé par GS1 Canada, a rassemblé les parties prenantes essentielles dans l'intention de cerner les lacunes des systèmes et des processus actuels pour la surveillance des pénuries de médicaments, de proposer les caractéristiques indispensables d'un système national unique et d'élaborer un plan de mise en œuvre. Rx\&D et l'ACMG se sont engagées à contribuer $100000 \$$ chacune afin de créer ce système national.
Entretemps, Rx\&D a ouvert sa plateforme pour la divulgation des pénuries de médicaments aux compagnies membres de l'ACMG et aux autres fabricants canadiens de médicaments. Sa plateforme bilingue est maintenant connue sous le nom de "base de données canadienne sur les pénuries de médicaments " et est accessible publiquement à www.penuriesdemedicaments.ca. À la fin de juillet, Santé Canada et Alberta Health (le ministère de la Santé albertain à la tête du Groupe de travail provincial et territorial sur les pénuries de médicaments) se sont interposés et ont invité la SCPH, l'ACGAP, l'ACCP, l'ACMG, l'APhC, l'AMC, BIOTECanada et Rx\&D à se joindre à eux ainsi qu'à l'Agence canadienne des médicaments et des technologies de la santé et aux compagnies d'achats groupés HealthPro et Medbuy pour former le Comité directeur multilatéral sur les pénuries de médicaments et exécuter des numéros collaboratifs sur la prévention et l'atténuation des pénuries partout au Canada.

La SCPH a exécuté un pas deux avec sa section de la Colombie-Britannique pour sensibiliser le public aux conséquences redoutées sur le système de santé si le gouvernement provincial mettait en application son plan d'élimination de la majoration salariale à la valeur du marché accordée aux pharmaciens d'établissements. Le duo a aussi effectué un mouvement impressionnant : la SCPH soulevant la gracieuse vidéo sur la valeur des pharmaciens d'établissements produite par la section de la C.-B. et la portant au conseil de la SCPH. La vidéo est maintenant à la disposition de toutes les sections (www.cshp.ca/advocacy/index_e.asp) et peut servir à mettre en valeur les pharmaciens d'établissements auprès du public, des politiciens et des fonctionnaires.

Le Conseil canadien de la résidence en pharmacie d'hôpital a entraîné les congressistes de la Conférence sur la pratique professionnelle de 2012 dans une valse à l'occasion de son $50^{\mathrm{e}}$ anniversaire. En 1956, Isabel Stauffer a proposé que la Conférence canadienne des facultés de pharmacie soit la cavalière de la SCPH pour instaurer les normes minimales relatives aux internats en pharmacie dans les hôpitaux. Cette idée s'est concrétisée en 1962 lorsque Isabel Stauffer, Orest Buchko, Phyllis Yagi, André Archambault et Glen Moir ont constitué le premier Conseil de la résidence. Vous pouvez visionner le diaporama retraçant les faits marquants de l'histoire du Conseil à www.cshp.ca/programs/residencyTraining/CHPRBinfo_e.asp. 
Quant à elle, la Fondation de la SCPH a chorégraphié de nouvelles occasions de bourses pour l'éducation très rock 'n' roll qui s'agenceront à son vénérable programme de bourses pour la recherche. La Fondation a ainsi inauguré à l'automne deux concours pour des approches novatrices de formation professionnelle, de dialogue et de transmission des connaissances qui concourent à améliorer la prestation des soins aux patients dans les établissements de santé et les milieux de soins de santé en collaboration. Le premier concours offre un financement pour des programmes de formation tels que stages, conférences thématiques et ressources pour les professionnels de la santé et les patients (www.cshpfoundation.ca/web/la/en/pa/ 6486eac590b14c21b850e8b8174d2706/template.asp). Le deuxième octroiera une bourse d'études à un leader de la pharmacie hospitalière en poste ou en puissance qui s'enrôlera dans la Pharmacy Leadership Academy de la Research and Education Foundation de l'American Society of Health-System Pharmacists, un programme de cyberapprentissage d'une durée de douze mois visant à rendre le leadership plus efficace et à former de futurs leaders en pharmacie hospitalière (www.cshpfoundation.ca/ web/la/en/pa/8D2A40F30B8A4883AC11DBA30BD18BBB/ template.asp).

La SCPH s'est aussi produite avec plusieurs autres organisations nationales pour une danse carrée : Agrément Canada, qui a sollicité des modifications à ses normes sur la gestion des médicaments et à ses pratiques organisationnelles requises; le Conseil canadien de l'agrément des programmes de pharmacie, qui a demandé les avis sur la deuxième ébauche des Normes et directives pour l'agrément des programmes de formation professionnelle de premier cycle en pharmacie; Santé Canada, qui a invité à commenter le projet de Règlement sur les nouvelles catégories de praticiens, destiné à désigner les sages-femmes, les infirmiers praticiens et les podiatres comme praticiens au sens de la Loi réglementant certaines drogues et autres substances, et le Résumé de l'étude d'impact de la réglementation connexe; et l'Association nationale des organismes de réglementation de la pharmacie, qui a consulté l'opinion sur son rapport préliminaire intitulé Pharmacy Practice Management Systems: Requirements to Support NAPRA Standards of Practice et la compétence supplémentaire proposée relativement à l'administration d'injections par les pharmaciens canadiens.

La SCPH s'est également laissée emporter par le rythme enlevant des mariachi et la musique langoureuse du tango. À titre de directrice générale de la SCPH, j’ai prononcé une conférence sur le rôle du pharmacien d'établissement au sein de l'équipe de soins interprofessionelle au Canada dans le cadre du deuxième congrès de pharmacie hospitalière de l'Asociación
Mexicana de Farmacéuticos de Hospital, à Puebla au Mexique en juin. Puis en novembre, j'ai fait un exposé sur un sujet analogue durant le premier congrès sud-américain de pharmacie hospitalière, organisé par l'Asociación Argentina de Farmacéuticos de Hospital, à Mar del Plata en Argentine.

Maints autres accomplissements ont prouvé que les employées de la SCPH savent sur quel pied danser. Les réseaux de spécialistes en pharmacie ont émigré de la plateforme Yahoo! Groupes vers le système de gestion d'association de la SCPH. Les listes de diffusion électronique de la Société, nommées eForums, font maintenant interface directement avec le répertoire des membres en ligne et résident sur le site Web de la SCPH. Pour la deuxième année consécutive, la SCPH s'est unie au comité organisateur de la Conférence de pharmacie Harrison en vue de tenir la $21^{\mathrm{e}}$ édition de l'activité de formation et de réseautage pour les leaders de pharmacie hospitalière, maintenant connue sous le nom de Séminaire de gestion en pharmacie de la SCPH (www.harrisonconference.ca). Nous avons ajouté deux publications à notre collection : Lignes directrices : déclaration et prévention des incidents médicamenteux, mises à jour par le comité directeur des normes de pratique, et les Evidence-Based Clinical Guidelines for Immigrants and Refugees, rédigées par la Canadian Collaboration for Immigrant and Refugee Health et sanctionnées par le conseil de la SCPH. En outre, le CSHP e-Newsbrief a été mis en circulation en avril; ce bulletin hebdomadaire rassemble les gros titres, les résultats de recherches et d'autres articles en ligne qui intéressent les pharmaciens d'établissements.

La troupe de danse de la SCPH s'est modifiée pendant la dernière année. Nous avons dit adieu à Amanda Cuirrier, agente par intérim du service aux membres et des prix, et nous avons souhaité la bienvenue à Cheryl Mallory au sein de ce même poste. Nous avons accueilli Pamela Saunders, qui s'est jointe à la troupe comme agente de bureau par intérim (y compris le soutien administratif au projet SCPH 2015 et au Conseil des associés).

J'espère que l'interprétation de la SCPH au cours de l'année 2012 a su vous impressionner et que nous avons gagné votre vote comme principal agent d'influence des progrès de la pratique en pharmacie hospitalière au Canada. Marchez sur nos pas comme bénévole et dansez en mesure avec nous!

Myrella Roy, B. Sc. Phm., Pharm. D., FCCP, est directrice générale de la SCPH. 